\title{
Correspondence
}

\section{A Note on the Wide-Band Gaussian Broadcast Channel}

\author{
ROBERT J. MCELIECE AND LAIF SWANSON
}

\begin{abstract}
Recently, Posner noted that on a wide-band Gaussian broadcast channel, ordinary time-shared coding performs almost as well as more sophisticated broadcast coding strategies. In this note, we shall give a quantitative version of Posner's result and argue that for certain realistic broadcast channels time sharing may suffice.
\end{abstract}

\section{INTRODUCTION}

In [4], Posner noted that on a wide-band Gaussian broadcast channel, ordinary time-shared coding performs almost as well as more sophisticated "broadcast coding." In this note, we shall investigate this interesting phenomenon a bit further and find a quantitative version of Posner's result. We shall find that for any discrete-time Gaussian broadcast channel for which the (symbol) signal-to-noise ratios (SNR's) are small, time-shared coding will never be at a serious disadvantage relative to broadcast coding. Since many high-performance communication systems do in fact operate at low (symbol) SNR's, this result is of significant practical importance, as we shall illustrate with a numerical example in Section III.

\section{The Gaussian Broadcast Channel; A Comparison of TIME-SHARED AND BROADCAST CODING}

In [1], Cover introduced a discrete-time memoryless channel model with one transmitter and two receivers, which he called a Gaussian broadcast channel. This channel has one input $X$ and two outputs $Y_{1}$ and $Y_{2}$, related by

$$
\begin{aligned}
& Y_{1}=X+Z_{1} \\
& Y_{2}=X+Z_{2}
\end{aligned}
$$

where $Z_{1}$ and $Z_{2}$ are independent mean zero Gaussian random variables, with variances $\sigma_{1}^{2}$ and $\sigma_{2}^{2}$. If the channel input $X$ is constrained "in mean power' by $E\left(X^{2}\right) \leqslant S$, then separately the two channels have capacities

$$
\begin{gathered}
C_{1}=\frac{1}{2} \log \left(1+\frac{S}{\sigma_{1}^{2}}\right) \\
C_{2}=\frac{1}{2} \log \left(1+\frac{S}{\sigma_{2}^{2}}\right) .
\end{gathered}
$$

To simplify the notation, we shall denote the first SNR $S / \sigma_{1}^{2}$ by $x_{1}$, the second SNR $S / \sigma_{2}^{2}$ by $x_{2}$, and the function $1 / 2 \log$ $(1+x)$ by $f(x)$. Then (3) and (4) become

$$
\begin{aligned}
& C_{1}=f\left(x_{1}\right) \\
& C_{2}=f\left(x_{2}\right) .
\end{aligned}
$$

Paper approved by the Editor for Coding Theory and Applications of the IEEE Communications Society. Manuscript received April 6, 1986; revised August 18, 1986. This work was carried out by the Jet Propulsion Laboratory, California Institute of Technology, under contract with the National Aeronautics and Space Administration and was supported in part by a grant from the Air Force Office of Scientific Research. The work of R. J. McEliece was supported in part by the Air Force Office of Scientific Research under Grant AFOSR-83-0296.

The authors are with the Department of Electrical Engineering and the Jet Propulsion Laboratory, California Institute of Technology, Pasadena, CA 91109.

IEEE Log Number 8613152.
We shall assume that the SNR for the first receiver is larger than that for the second receiver, i.e., that $x_{1}>x_{2}$, and call the first receiver the better receiver and the second receiver the weaker receiver.

A fundamental question about such a broadcast channel is this: suppose we wish to send certain information, called the common information simultaneously to both receivers. If we do this, how much extra information, called bonus information, can we send to the better receiver at the same time?

In the time-sharing approach to this problem, the transmitter dedicates a fixed fraction $1-\rho(0 \leqslant \rho \leqslant 1)$ of the total transmission time to sending the common information, which is coded for the weaker receiver, and so will be comprehensible to both receivers. By (4), during this common time, information can be transmitted at a maximum rate $C_{2}$. During the remaining fraction $\rho$ of the transmission time, the transmitter sends the bonus information, coded for the stronger receiver, at a maximum rate $C_{1}$. The bonus information is thus sent at a rate above the capacity of the weaker channel and so will not be comprehensible to the weaker receiver. Thus, for time-shared coding, the information rates will be

$$
\begin{gathered}
\text { Time-Shared Common Rate (TCR) }=(1-\rho) f\left(x_{2}\right) \\
\text { Time-Shared Bonus Rate (TBR) }=f\left(\rho x_{1}\right)
\end{gathered}
$$

where the parameter $\rho$ can be selected arbitrarily by the transmitter.

Cover showed in [1], however, that it is possible to do better than time sharing. Using a technique now called broadcast coding, he showed that for any choice of the parameter $\alpha$, $0 \leqslant \alpha \leqslant 1$, the following rates are in principle achievable:

$$
\begin{gathered}
\text { Broadcast Common Rate }(\mathrm{BCR})=f\left(x_{2}\right)-f\left(\alpha x_{2}\right) \\
\text { Broadcast Bonus Rate }(\mathrm{BBR})=f\left(\alpha x_{1}\right) .
\end{gathered}
$$

The two pairs of equations (5), (6) and (7), (8) give parametric descriptions of two curves, as shown in Fig. 1. It is a simple exercise in calculus to verify that, as suggested by Fig. 1, the broadcast coding curve always lies above the timeshared coding curve. El Gamal and Cover [2] showed that the region of the first quadrant bounded by the broadcast coding curve is the capacity region for the Gaussian broadcast channel, so no further improvement is possible.

We now come to our main result, which is simple to prove, but has surprising consequences.

Theorem: If $\alpha$ and $\rho$ are chosen so that the common rates in (5) and (7) are equal, then the bonus rates satisfy the following:

$$
\frac{\mathrm{BBR}}{\mathrm{TBR}} \leqslant \frac{x_{1}}{x_{2}} \frac{\log \left(1+x_{2}\right)}{\log \left(1+x_{1}\right)} .
$$

Proof: For the two common rates to be equal, we have, from (5) and (7), that

$$
f\left(\alpha x_{2}\right)=\rho f\left(x_{2}\right) .
$$

On the other hand, the ratio of the bonus rates is, from (6) and (8),

$$
\frac{f\left(\alpha x_{1}\right)}{\rho f\left(x_{1}\right)} .
$$




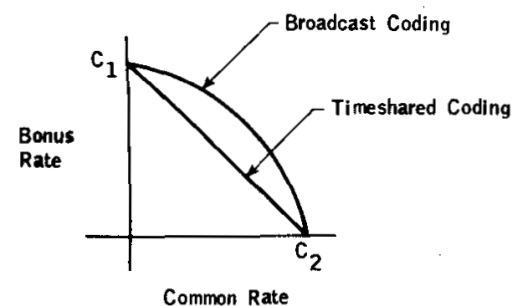

Fig. 1. The general shape of the broadcast coding and the time-shared coding curves.

Combining (9) and (10), we see that, for a fixed common rate, the ratio of the bonus rates is

$$
\frac{f\left(\alpha x_{1}\right)}{f\left(\alpha x_{2}\right)} \cdot \frac{f\left(x_{2}\right)}{f\left(x_{1}\right)} .
$$

The desired result now follows from the fact that the function $f\left(\alpha x_{1}\right) / f\left(\alpha x_{2}\right)$ is a decreasing function of $\alpha$ and approaches $x_{1} / x_{2}$ as $\alpha \rightarrow 0$.

Corollary: Since $\log \left(1+x_{2}\right)<x_{2} \log e$, we also have

$$
\frac{\mathrm{BBR}}{\mathrm{TBR}}<\frac{x_{1} \log e}{\log \left(1+x_{1}\right)}
$$

independent of $x_{2}$. Since $\log \left(1+x_{1}\right) \sim x_{1} \log e$ for small values of $x_{1}$, the corollary shows that the ratio BBR/TBR is very close to 1 whenever the better SNR is small. We will explore the implications of this in the next section.

\section{ApPlications}

The capacity formulas (3)-(8) apply only to discrete-time channel models. However, using standard techniques explained in [3, ch. 4], for example, these formulas can all be transformed into formulas that apply to continuous-time models. Indeed, if $B$ is the channel bandwidth (in hertz), $P$ is the average transmitter power (in watts), and if $N_{1}, N_{2}$ are the noise spectral densities (in watts per hertz) for the two receivers, the continuous-time analogs for $(3)-(8)$ are as follows:

$$
\begin{gathered}
C_{1}=B \log \left(1+\frac{P}{N_{1} B}\right) \\
C_{2}=B \log \left(1+\frac{P}{N_{2} B}\right) \\
\mathrm{TCR}=(1-\rho) B \log \left(1+\frac{P}{N_{2} B}\right) \\
\mathrm{TBR}=\rho B \log \left(1+\frac{P}{N_{1} B}\right) \\
\mathrm{BCR}=B \log \left(1+\frac{P}{N_{2} B}\right)-B \log \left(1+\alpha \frac{P}{N_{2} B}\right) \\
\mathrm{BBR}=B \log \left(1+\alpha \frac{P}{N_{1} B}\right) .
\end{gathered}
$$

For these channel models, the quantities $P / N_{1} B$ and $P / N_{2} B$ play the roles of the abstract SNR's $x_{1}$ and $x_{2}$ in the discretetime models, and the theorem of the last section implies that

$$
\frac{\mathrm{BBR}}{\mathrm{TBR}} \leqslant \frac{N_{2}}{N_{1}} \frac{\log \left(1+P / N_{2} B\right)}{\log \left(1+P / N_{1} B\right)}<\frac{\left(P / N_{1} B\right) \log e}{\log \left(1+P / N_{1} B\right)} .
$$

It follows from (12) that for any value of $P, N_{1}$, and $N_{2}$ (assuming $N_{2} \geqslant N_{1}$ ), for sufficiently large bandwidth, the ratio (BBR/TBR) will be very close to 1 . This is our quantitative version of the result cited by Posner.

As a numerical example, we compare the performance of broadcast coding and time-shared coding in a scenario similar to the Voyager 2 communications environment. For Voyager the Gaussian broadcast channel model is appropriate, with the two receivers corresponding to "good weather'" and 'bad weather" at the receiving stations [5]. Thus, in this application the two receivers are physically identical but correspond to different and unpredictable reception conditions. The common data are mission critical, whereas the bonus data are information the investigators expect to receive, but may in an extreme case have to live without. The maximum (good weather) Voyager data rate at Uranus is about $30 \mathrm{kbits} / \mathrm{s}$.

If we assume a transmission bandwidth of about $200 \mathrm{kHz}$ (thus allowing for a six- or seven-fold bandwidth expansion for coding), formula (3a) implies that for a capacity of 30 kbits/s, the quantity $P / N_{1} B$ should be at least equal to $2^{0.15}$ $-1=0.1096$. According to (12), this implies that independent of the capacity of the weaker channel, the maximum ratio of the BBR to the TBR is $\left(2^{0.15}-1\right) / \log \left(2^{0.15}\right)=1.054$. This corresponds to about $0.2 \mathrm{~dB}$. Even this small gain would be achievable only in the limit as the SNR on the "bad weather" channel approached zero. In one Voyager scenario [5], it is assumed that bad weather attenuation (the so-called "99' percent weather" condition) is about $4 \mathrm{~dB}$. In this case, $P / N_{2} B=(0.398) \times P / N_{1} B$, and by $(12),(\mathrm{BBR}) /(\mathrm{TBR})$ $<1.0352$, or about $0.15 \mathrm{~dB}$. In either case, we conclude that, at least in this one practical situation, broadcast coding offers no significant advantage over time sharing.

\section{REFERENCES}

[1] T. M. Cover, "Broadcast channels," IEEE Trans. Inform. Theory: vol. IT-11, pp. 2-14, 1972.

[2] A. El Gamal and T. M. Cover, "Multiple user information theory," Proc. IEEE, vol. 68, pp. 1466-1483, 1980.

[3] R. J. McEliece, The Theory of Information and Coding. Reading, MA: Addison-Wesley, 1977.

[4] E. C. Posner, "Strategies for weather-dependent data acquisition," IEEE Trans. Commun., vol. COM-31, pp. 509-517, 1983.

[5] L. Swanson and J. H. Yuen, "A strategy for successful deep space information transmission in bad weather," Jet Propulsion Lab., Calif. Inst. Technol., Pasadena, CA, TDA Progr. Rep. vol. 42-78, p. 151, 1984.

\section{On the Mean Number of Stuffing Bits in a Prefix-Synchronized Code}

\author{
STAVROS PAPASTAVRIDIS
}

\begin{abstract}
Artom [1] suggested a bit-stuffing technique to map data blocks into prefix-synchronized code words. In this correspondence we compute the mean number of stuffing bits required. We prove that the average number of stuffing bits per block is quite small.
\end{abstract}

Paper approved by the Editor for Coding Theory and Applications of the IEEE Communications Society. Manuscript received December 12, 1985; revised June 19, 1986 and July 14, 1986.

The author is with the Applied Mathematics Division, University of Patras, 26110 Patras, Greece.

IEEE Log Number 8613160. 